\title{
ANDES

\section{Factores de riesgo asociados a complicaciones de catéteres centrales de inserción periférica en recién nacidos}

\author{
Risk factors associated with complications of peripherally inserted central catheter \\ in newborn infants
}

\author{
Miriam Faunes Pérez ${ }^{a}$ Álvaro Gonzalez Morandéb, María Eugenia Pérez Arriarána, \\ Verónica Torres Tapia ${ }^{\mathrm{a}}$, Mónica Avaca Bengochea ${ }^{\mathrm{a}}$, Marta Alcaide Aracena ${ }^{\mathrm{a}}$, \\ Patricia Avalos Odano ${ }^{a}$, Soledad Urzúa Baquedano ${ }^{b}$, Jorge Fabres Biggs ${ }^{b}$
}

\author{
aEnfermera-Matrona. Servicio de Neonatología, Hospital Clínico Red de Salud UC-CHRISTUS, Escuela de Medicina, Pontificia Universidad Católica \\ de Chile. Santiago, Chile \\ bDepartamento de Neonatología, División de Pediatría, Escuela de Medicina, Pontificia Universidad Católica de Chile. Santiago, Chile
}

Recibido: 25 de noviembre de 2020; Aceptado: 18 de marzo de 2021

\section{¿Qué se sabe del tema que trata este estudio?}

Los catéteres venosos centrales insertados periféricamente permiten accesos confiables y de mayor duración en neonatos, sin embargo en ocasiones presentan complicaciones mayores como infecciones y trombosis. Estas se asocian con menor edad gestacional y uso prolongado

\begin{abstract}
¿Qué aporta este estudio a lo ya conocido?
Un $4 \%$ presentó complicaciones mayores destacando infecciones y derrames por extravasación. Se confirma su asociación con $<$ EG y uso $>14$ días. Las infecciones se asociaron con $>$ número de punciones, y las no infecciosas, con uso de extremidades superiores y malposición.
\end{abstract}

\section{Resumen}

Objetivo: Describir la evolución y complicaciones asociadas al uso del catéter venoso central de inserción periférica en recién nacidos e identificar los factores de riesgo asociados a ellas. Sujetos y Método: Estudio analítico del seguimiento de catéteres instalados en 541 recién nacidos hospitalizados en una Unidad Neonatal. Se caracterizan su evolución y complicaciones. Para evaluar los factores de riesgo asociados a complicaciones se realizó análisis de regresión logística multivariado. Resultados: Se instalaron 655 catéteres en 541 recién nacidos con peso de nacimiento entre 420 y 4.575 g. La duración promedio fue de $11,6 \pm 8,5$ días. En 29 pacientes $(4,4 \%)$ se observaron complicaciones mayores, siendo la más frecuente la infección del torrente sanguíneo $(n=17)$, determinando una tasa de 2,25 \%o días-catéter. Ellas fueron más frecuentes en aquellos catéteres que permanecieron $>14$ días: $9 / 179(5 \%)$ vs 8/476 $(1,7 \%)$ de los $\leq 14$ días $(\mathrm{p}<0,05)$. Entre las complicaciones no infecciosas destacaron: derrame pleural por extravasación $(n=6)$ y trombosis auricular $(n=3)$. El
Palabras clave: Catéteres Percutáneos; Catéter Venoso Central; Recién Nacido; Complicaciones 
análisis multivariado mostró que ellas se asociaron a una edad gestacional $<28$ semanas: OR 5,9 (IC95\%: 1,2-40) y a su instalación en extremidades superiores OR 3,2 (IC95\% 1,1-7,0). Las infecciones se asociaron a $>$ número de punciones en su instalación: OR 2,1 (IC95\% 1,2-4,8) por punción y edad gestacional < 28 semanas: OR 7,9 (IC 95\%: 1,4-73). Conclusión: Los catéteres resultaron de larga duración y baja tasa de complicaciones mayores. Éstas se presentan mayormente en prematuros extremos. Las infecciones se asociaron a > número de punciones y duración > 14 días, las otras complicaciones se asociaron con la instalación en extremidades superiores.
Keywords:

Percutaneous

Catheters;

Central Venous

Catheters;

Newborn;

Complications

\section{Introducción}

En los últimos años, debido al avance del cuidado intensivo neonatal, los recién nacidos de muy bajo peso (RNMBP) y aquellos con patologías graves y/o malformaciones complejas han tenido una importante mejoría de su sobrevida ${ }^{1-3}$. Su estadía hospitalaria puede ser prolongada requiriendo terapias complejas y accesos venosos por mayor tiempo. Es por ello que se han propuesto distintas técnicas para tener accesos venosos de mejor calidad y permanencia ${ }^{4,5}$.

Los catéteres centrales se han utilizado en neonatología desde hace varias décadas, sin embargo, su uso prolongado se ha asociado a infecciones y otras complicaciones $^{6-8}$. Entre las técnicas alternativas destaca la instalación percutánea de catéteres de silicona a través de una vena periférica, conocido como cateterismo venoso central de inserción periférica (CVCIP) o "catéter percutáneo". Éste es utilizado en RN que requieren tratamientos endovenosos por períodos mayores a 7 días. Experiencias nacionales e internacionales revelan varias ventajas como: menor traumatismo en su instalación, mayor duración y confiabilidad de los acce$\operatorname{sos}^{9-13}$. La instalación es relativamente fácil, pudiendo ser realizado en la unidad del paciente. Sin embargo, se han descrito complicaciones con su uso, tanto mayores (con riesgo vital), como menores. Entre las primeras, destacan: infecciones del torrente sanguíneo (ITS), arritmias, derrames pericárdico y pleural ${ }^{14-18}$. Entre las complicaciones menores destacan la flebitis, infiltración/extravasación de fluidos y obstrucción ${ }^{10,12,18}$.

El objetivo del presente estudio es describir la evolución y complicaciones del uso de CVCIP en una unidad de cuidado intensivo neonatal terciaria e identificar los factores de riesgo asociados a la presencia de complicaciones mayores.

\section{Sujetos y Método}

Estudio analítico y retrospectivo, en base a información recolectada prospectivamente para el seguimiento de los CVCIP instalados en el Servicio de Neonatología del Hospital Clínico de la Red de Salud UC-CHRISTUS, entre enero de 1998 y diciembre del 2014. Se incluyeron RN hospitalizados que requirieron la instalación de un CVCIP. Se excluyeron del análisis, aquellos con un CVCIP instalado en otro servicio $\mathrm{y}$ aquellos que fueron trasladados hacia otros centros con un CVCIP in situ. 
Los catéteres utilizados fueron de silicona de 1 ó 2 French (24 a 28 Gauge), radiopacos, con orificio terminal único recto, de 30 a $40 \mathrm{~cm}$ de largo. La instalación fue realizada en la unidad del paciente y la ubicación de la punta del catéter fue controlada radiológicamente. Se instruyó que cuando el catéter fuera insertado desde una extremidad superior, la punta debería estar en la vena cava superior (VCS) o en la unión de la VCS y la aurícula derecha. Cuando fuera insertado desde una extremidad inferior, la punta del catéter debería estar por encima de las vértebras L4-L5, pero no en el corazón. Luego del control radiográfico, se fijó con cinta adhesiva estéril (Steri-strip ${ }^{\circledR}$ ) a $1 \mathrm{~cm}$ del sitio de inserción. La parte del catéter sobrante fuera del sitio de inserción se fijó con la misma cinta enrollado sobre la piel y se cubrió con apósito adhesivo transparente (Tegaderm ${ }^{\circledR}$ ). El sitio de inserción se cubrió con una gasa estéril de $1 \times 1 \mathrm{~cm}$ y fijó con cinta adhesiva también estéril. Se realizó seguimiento con observación diaria y retiro según protocolo estandarizado del servicio9. Se realizaron curaciones cada $48 \mathrm{~h}$ utilizando clorhexidina alcohólica al 0,5\% y apósito de gasa. Los CVCIP se mantuvieron con una infusión de base ya sea nutrición parenteral o solución glucosada con electrolitos con la adición de heparina $(0,5-1 \mathrm{UI} / \mathrm{ml})$.

Se utilizó una hoja estandarizada de recolección de datos, la cual se completó en forma prospectiva durante la hospitalización del paciente. Las variables incluidas en el registro fueron: peso de nacimiento, sexo, edad gestacional (EG), Apgar, diagnósticos de ingreso, edad de instalación, número de punciones, lugar de inserción, ubicación de la punta, días de permanencia y causa de retiro de catéter. Se definió "larga duración" aquellos que permanecieron por más de 7 días. También se registraron las posibles complicaciones mayores: sepsis, derrame pleural o pericárdico, cateterización inadvertida de vasos no habituales, trombosis en aurícula o vasos mayores; y complicaciones menores como flebitis, infiltración/extravasación, obstrucción, desplazamiento o retiro involuntario y sección del catéter. Se definió como ITS asociada al catéter: la presencia de al menos un hemocultivo periférico (+) asociado a deterioro clínico mientras el paciente tenía el catéter in situ y ausencia de evidencias de infección en otro sitio.

\section{Análisis estadístico}

Para el análisis de los datos se utilizó el Programa JMP 9 (Instituto SAS, NC, EE.UU.). Para la descripción de resultados se usaron medidas de tendencia central y dispersión. Para las comparaciones de variables continuas, se usaron las pruebas "t" de Student o MannWhitney. Las variables categóricas fueron comparadas con el test de $\mathrm{Chi}^{2} \mathrm{y}$, si el número de observaciones era $<5$, se utilizó el test exacto de Fisher. Se consideró sig- nificativo un valor $\mathrm{p}<0,05$. Para identificar y ponderar los factores de riesgo asociados a complicaciones mayores se realizó análisis de regresión logística multivariado. Se realizaron dos modelos, uno para evaluar factores asociados a complicaciones no infecciosas y un segundo modelo para analizar los factores asociados a la incidencia de infección relacionada con el catéter.

Este estudio fue aprobado por el Comité Ético Científico de Ciencias de la Salud de la Universidad Católica con dispensa de consentimiento informado, dado que los datos son de un procedimiento estándar en el cuidado intensivo neonatal, que fueron recolectados desde los registros internos del Servicio y traspasados a una base de datos anónima, sin la identificación de los pacientes involucrados.

\section{Resultados}

Se estudiaron 655 catéteres instalados en $541 \mathrm{RN}$, la duración promedio fue de $11,6 \pm 8,5$ días, con un rango de 1 a 64 días, 429 (65\%) de ellos permanecieron en forma prolongada por más de 7 días. Esto hizo un total de 7.539 días catéter. La EG promedio de los niños fue de $31,3 \pm 4,7$ semanas, con un rango entre 23 y 41 semanas. Su peso de nacimiento promedio fue $1.698 \pm 984$ g, con un rango entre 420 g y 4.575 g. Entre los diagnósticos más frecuentes de los $\mathrm{RN}$ que requirieron CVCIP están RNMBP: 302 (56 \%), hipertensión pulmonar persistente 78 (14\%), hernia diafragmática $48(9 \%)$, cardiopatías congénitas $32(6 \%)$ y otras malformaciones gastro-intestinales $28(5 \%)$.

Del total de catéteres instalados, el $44,9 \%$ se instaló al primer intento, con un promedio de 2,2 $\pm 1,4$ punciones, con un rango de 1 a 7 punciones. El sitio de inserción más frecuente correspondió a las extremidades superiores en un $80,1 \%$ (venas del pliegue del codo, mano, antebrazo y axila), prefiriéndose el lado derecho en un 56\% de ellas. Un 15,9\% se instaló en extremidades inferiores y un $4,0 \%$ en venas del cuello. El motivo de retiro más frecuente fue el término de tratamiento $(60,2 \%)$.

Las complicaciones mayores se presentaron en 29 catéteres $(4,4 \%)$, siendo la más frecuente la ITS, en 17 $(2,6 \%)$, lo que determinó una tasa de infección de 2,25 $\%$ días-catéter (tabla 1). Los microorganismos aislados en ITS asociada a catéter fueron: Staphylococcus coagulasa negativo (7), Candida albicans (6), Pseudomona aureginosa (1), Klebsiella oxytoca (1), Staphylococcus aureus (1) y Enterococus fecalis (1), un paciente presentó co-infección a 2 agentes: Staphylococcus epidermidis $y$ Candida. Como se observa en la tabla 2, la infección asociada a catéter se presentó más frecuentemente en los prematuros de extremo bajo peso $(<1.000 \mathrm{~g})$, con una tasa de 4,98 por 1.000 días catéter. Como se obser- 
Tabla 1. Complicaciones observadas en CVCIP en este grupo RN

\begin{tabular}{llcc}
\hline & Complicación & $\mathrm{n}$ & $\%$ \\
\hline Complicaciones mayores & ITS/CVC & 17 & $2,6 \%$ \\
$(29=4,4 \%)$ & Derrame pleural & 6 & $1,2 \%$ \\
& Derrame pericárdico & 1 & $0,15 \%$ \\
& Trombosis en aurícula & 3 & $0,5 \%$ \\
& Desplazamiento hacia columna (epidural) o retroperitoneo & 2 & $0,3 \%$ \\
Complicaciones menores & Flebitis & 69 & $10,5 \%$ \\
$(182=27,8 \%)$ & Obstrucción & 46 & $7,0 \%$ \\
& Infiltración/extravasación edema extremidad & 44 & $6,7 \%$ \\
& Desplazamiento o retiro involuntario & 17 & $2,6 \%$ \\
& Sección del catéter & 6 & $0,9 \%$ \\
\hline
\end{tabular}

ITS: Infección Tracto Sanguíneo; CVC: catéter venoso central.

Tabla 2. Relación entre el peso de nacimiento e infección

\begin{tabular}{lcccc}
\hline Categoría de peso & $<1.000 \mathrm{~g}$ & $1.001-1.500 \mathrm{~g}$ & $>1.500 \mathrm{~g}$ & Total \\
\hline $\mathrm{N}^{\circ}$ catéteres & 201 & 175 & 279 & 655 \\
$\mathrm{~N}^{\circ}$ días catéteres & 2.009 & 1.676 & 3.747 & 7.539 \\
ITS: $\mathrm{n}(\%)$ & $10(5,0 \%)$ & $3(1,7 \%)$ & $4(1,4 \%)$ & $17(2,6 \%)$ \\
Tasa / 1.000 días cat. & 4,98 & 1,79 & 1,07 & 2,25 \\
\hline
\end{tabular}

Tasa Minsal: 3,41\% días catéter en pacientes pediátricos (2012). ITS: Infección tracto sanguíneo.

Tabla 3. Relación entre duración del catéter con infección (x semana)

\begin{tabular}{lccccc}
\hline Duración catéter (días) & $0-7$ & $8-14$ & $15-21$ & $>21$ & Total \\
\hline$N^{\circ}$ total catéteres & 655 & 429 & 179 & 67 & 655 \\
$N^{\circ}$ días catéter & 3.899 & 5.381 & 3.413 & 2.010 & 7.539 \\
ITS $(\mathrm{n})$ & 3 & 5 & 7 & 2 & 17 \\
Tasa $\%$ & $0,5 \%$ & $1,1 \%$ & $3,9 \%$ & $3,0 \%$ & $2,6 \%$ \\
Tasa / 1.000 días cat. & 0,7 & 0,93 & 2,1 & $2,25 \% 0$ \\
\hline
\end{tabular}

ITS: Infección tracto sanguíneo.

va en la tabla 3, el riesgo de infección también aumentó con la duración del catéter, en especial en aquellos catéteres que permanecieron $>14$ días donde 9/179 (5\%) presentaron ITS, lo que fue significativamente mayor a los $8 / 476(1,7 \%)$ de los catéteres que permanecieron $\leq 14$ días $(\mathrm{p}<0,05)$. Sin embargo, tuvimos varios catéteres que permanecieron $>21$ días sin infección, incluso la tasa bajó después de la tercera semana. En el análisis multivariado la presencia de ITS asociada a catéter se asoció a una EG menor de 28 semanas y a un mayor número de punciones durante su instalación (tabla 4). Se encontró también una tendencia a aumentar el riesgo con la duración del catéter: OR 1,9 (IC 95\% 0,9-5,1) por cada semana adicional $(\mathrm{p}=0,1)$.
Entre las complicaciones mayores no infecciosas observadas destacaron el desarrollo de colecciones líquidas por extravasación en: espacio pleural $(n=6)$ o pericárdico $(n=1)$, y la presencia de trombosis en aurícula derecha $(\mathrm{n}=3)$. Uno de los prematuros extremos falleció como consecuencia de deterioro respiratorio progresivo luego de una extravasación con derrame pleural masivo. Los otros 6 casos de derrames por extravasación pudieron manejarse con retiro del catéter, drenaje de la colección y aumento transitorio de su terapia respiratoria. En 6 de los 7 pacientes que presentaron derrames se evidenció una mala posición de la punta del catéter, ya sea al momento de su instalación o porque se había desplazado posteriormente. En 
Tabla 4. Factores de Riesgo para desarrollar Sepsis asociada a Catéter. Odds Ratios y sus Intervalos de confianza del $95 \%$

\begin{tabular}{lccc}
\hline Condición & Odds Ratio & Intervalos confianza 95\% & Valor $p$ \\
\hline Peso al nacer (por 100 g $\downarrow$ ) & 1,3 & $0,3-3,7$ & 0,3 \\
Edad gestacional $<28 \mathrm{sem}$ & 7,9 & $1,4-73$ & $<0,03$ \\
Sexo masculino & 2,1 & $0,6-8,7$ & 0,25 \\
Apgar 5 min $\leq 5$ & 1,8 & $0,2-8,8$ & 0,51 \\
$N^{\circ}$ punciones (x c/punción) & 2,1 & $1,3-4,8$ & $<0,01$ \\
Ubicación extremidad superior & 2,2 & $0,4-9,1$ & 0,3 \\
Duración catéter (por semana $\uparrow$ ) & 1,9 & $0,9-5,1$ & 0,1 \\
\hline
\end{tabular}

Tabla 5. Factores de Riesgo para desarrollar Complicaciones Mayores. Odds Ratios y sus Intervalos de Confianza del $95 \%$

\begin{tabular}{lccc}
\hline Condición & Odds Ratio & Intervalos Confianza 95\% & Valor $p$ \\
\hline Peso al nacer (por 100 g $\downarrow$ ) & 1,4 & $0,4-4,7$ & 0,26 \\
Edad gestacional < 28 sem & 5,9 & $1,2-40$ & $<0,04$ \\
Sexo Masculino & 1,4 & $0,4-4,2$ & 0,5 \\
Apgar 5 min $\leq 5$ & 1,2 & $0,2-5,2$ & 0,8 \\
Ubicación Extremidad Superior & 3,2 & $1,1-9,7$ & $<0,05$ \\
Duración Catéter (por semana $\uparrow$ ) & 1,7 & $0,5-7,3$ & 0,4 \\
\hline
\end{tabular}

5 de ellos, la punta del catéter estaba en la vena subclavia sin alcanzar la VCS. El análisis multivariado mostró que estas complicaciones mayores no infecciosas se asociaron significativamente a una $\mathrm{EG}<28$ semanas y a su instalación en extremidades superiores, quienes tuvieron un riesgo 3 veces mayor de desarrollarlas que aquellos instalados en las extremidades inferiores (tabla 5).

Las complicaciones menores se encontraron en un 27,4\% del total de los catéteres (tabla 1), siendo la más frecuentes la flebitis en un 10,5\% y la obstrucción en un $7,0 \%$.

\section{Discusión}

En nuestro Servicio de Neonatología se instalan CVCIP desde la década de los 90, siendo un procedimiento habitual del cuidado intensivo neonatal. En la presente investigación destacamos que el uso de CVCIP resultó de larga duración y con una baja tasa de complicaciones mayores. La presencia de ellas se asoció en forma significativa a una EG $<28$ semanas, y para el desarrollo de una ITS, a un mayor número de punciones durante su instalación y una duración mayor de 2 semanas. Por otra parte, la instalación en extremidades inferiores se asoció a un menor riesgo de complicaciones no infecciosas.
La complicación mayor más frecuentemente observada en la muestra fue la ITS, resultando en una tasa de 2,25 por 1.000 días catéter. Si bien esta tasa es menor que la tasa observada por el Ministerio de Salud de Chile para pacientes pediátricos en el año $2012(3,41 \text { por } 1.000 \text { días catéter })^{19}$, no hay estándares nacionales específicos para CVCIP en RN, ni tampoco desagregados por EG o peso de nacimiento. Sólo existen estándares para catéteres umbilicales. La tasa reportada en este estudio es similar a reportes internacionales recientes ${ }^{15,20-24}$. En este estudio identificamos que los factores de riesgo asociados a una mayor incidencia de ITS fueron una EG $<28$ semanas y un mayor número de punciones durante la instalación del catéter. Muchos autores han descrito la asociación entre infección y menor EG, especialmente en los prematuros $<1.000 \mathrm{~g}$, debido a la deficiencia en su sistema inmunitario y los requerimientos de terapias invasivas prolongadas ${ }^{21-23}$.

En relación al número de punciones durante la inserción, encontramos que por cada punción adicional el riesgo de infección se incrementó en 2,1 veces, esta es una importante observación que ha sido evaluada en muy pocos estudios. Njere y cols., no encontraron relación entre la frecuencia de infección y el número de intentos de inserción, pero en dicho reporte eran pocos niños y con una tasa muy alta de infección ${ }^{25}$. En este 
estudio el número de punciones fue minuciosamente registrado y claramente el riesgo de ITS aumentó con el mayor número de intentos. Por ello es muy importante extremar las medidas de higiene durante su instalación, y si se fracasó en un primer intento, usar nuevos equipos y paños estériles, e higienizar bien el área antes de realizar una nueva punción. Similar a otros reportes, un mayor número de días de permanencia del catéter se relacionó con una mayor tasa de infección, en especial si permanecían más de 14 días ${ }^{20-22}$. Sin embargo, tuvimos un número importante de catéteres que duraron más de 21 días sin infección, incluso la tasa bajó discretamente después de la tercera semana, esto hizo que en el análisis multivariado el aumento del riesgo por semana de duración tuviera menos impacto. Esto es similar a lo reportado por Milstone y cols, en una de las series más grandes de seguimiento de CVCIP y su relación con infección en $\mathrm{RN}^{21}$. Ellos describen un aumento importante del riesgo de ITS con la duración del catéter los primeros 14 días, luego de lo cual la tasa de infección si bien permanecía alta, aumentaba en menor proporción. Entre los factores que pudieran explicar esta diferencia con otros reportes es la menor intervención y práctica de curaciones realizadas en nuestro servicio. El microorganismo más frecuente correspondió a Staphylococcus coagulasa negativo, coincidiendo con lo reportado por otros ${ }^{21-23}$. El segundo microorganismo fue Candida albicans que encontramos principalmente en prematuros extremos y algunos RN con malformaciones que requirieron procedimientos quirúrgicos complejos. Interesantemente, las sepsis por hongos disminuyeron y casi desaparecieron en el segundo período (desde el año 2009 en adelante), lo que se asoció a restricción del uso de antibióticos de amplio espectro en el servicio ${ }^{26}$.

Entre las complicaciones mayores no infecciosas destacó el desarrollo de colecciones líquidas por extravasación en el espacio pleural o pericárdico, y la trombosis auricular. Éstas se relacionaron a una inadecuada ubicación de la punta del catéter, lo que se ha descrito también en otras publicaciones ${ }^{16-20}$. Por ello, es prioritario realizar una adecuada medición de la longitud a introducir y controlar radiológicamente la ubicación de la punta luego de su inserción con dos proyecciones: antero-posterior y lateral. También es muy importante re-controlar siempre que el catéter sea movilizado para su reubicación o se haya desplazado de su fijación original. La posición en la vena subclavia no debe considerarse apropiada, como vimos en esta serie 5 de los 7 casos de derrame se asociaron a esta posición. Es muy importante también contar con catéteres radiopacos que permiten su mejor visualización. El uso de ultrasonido es una herramienta útil tanto para la inserción de catéteres centrales como para certificación de su ubicación ${ }^{27}$. También hay que considerar que los catéteres pueden desplazarse o migrar, por lo que es importante re-evaluar su posición en controles sucesivos, sugerimos al menos cada 7 días si el catéter permanece por tiempos prolongados. En esta serie también observamos desplazamientos hacia retroperitoneo, y en un paciente hacia el espacio epidural. Estos correspondieron a catéteres insertados en las extremidades inferiores, y si bien esto es infrecuente, se ha descrito anteriormente ${ }^{28,29}$. Dos proyecciones radiográficas (anteroposterior y lateral) ayudan a confirmar la posición. En los catéteres instalados por extremidades inferiores la punta puede estar inadvertidamente en una vena lumbar ascendente y puede parecer en buena posición en la proyección anteroposterior ${ }^{28}$.

Interesantemente en este estudio observamos una menor frecuencia de complicaciones graves no infecciosas en aquellos catéteres instalados en extremidades inferiores. Varios estudios han mostrado mayor incidencia de complicaciones con los CVCIP de las extremidades superiores, lo que se ha relacionado a una mayor frecuencia de malposición de la punta ${ }^{17,18,30,31}$.

Entre las complicaciones menores destacó la flebitis, que se presentó en 10,5\% de los casos, similar a lo reportado en otras series ${ }^{15,20}$. Ésta se observó más frecuentemente en pacientes más pequeños, la mayoría fue transitoria y no se asoció a infección. El tamaño de la vena en relación al diámetro del catéter es uno de los factores que podría incidir en su aparición. Se describe también flebitis química en relación al talco de los guantes usados en la instalación, recomendándose el uso de guantes sin talco ${ }^{13}$. El tipo de material del catéter y el movimiento de éste dentro del vaso pueden causar irritación, por lo que una adecuada inmovilización y el uso de catéteres de silicona contribuirían a disminuir la flebitis ${ }^{9,20}$. La oclusión del catéter se presentó en un 6,9\%, tasa más baja que la publicada en otros estudios ${ }^{14,20}$. En nuestra práctica es habitual el adicionar heparina a las soluciones infundidas $(0,5-1 \mathrm{UI} \times \mathrm{ml})^{32,33}$. Tampoco se realiza toma de exámenes ni administración de transfusiones sanguíneas a través de ellos, recomendación para los catéteres $\leq 2$ French $^{13}$.

Entre las fortalezas de esta investigación, destacan el gran tamaño de la muestra y variedad de $\mathrm{RN}$ reclutados, lo que permite mostrar la incidencia de complicaciones e identificar mejor los factores de riesgo asociados. Entre sus debilidades están: no tener el seguimiento de los RN que se trasladaron con CVCIP a otros centros y de no tener el registro en los cuales la instalación fue frustra. Por otra parte, durante el largo período del estudio (15 años), hubo cambio en algunas prácticas que pudieron afectar los resultados, como lo fue el uso de antibióticos en la unidad. La incorporación de "bundles" (paquete de medidas) para disminuir infecciones en el servicio, que incluyó la res- 
tricción del uso de antibióticos de amplio espectro en los últimos años, pudo influir en la menor incidencia de ITS observada ${ }^{26}$. Entre estas medidas destacamos también, el insistir en realizar un muy buen lavado con jabón de clorhexidina y pincelado posterior con clorhexidina alcohólica antes de la inserción.

En conclusión, el uso de CVCIP en este grupo de $\mathrm{RN}$ resultó en una larga duración de ellos y con una baja tasa de complicaciones mayores. Éstas se presentan mayormente en prematuros extremos. Las ITS se asociaron a un mayor número de punciones y duración de más de 14 días, las otras complicaciones se observaron más frecuentemente asociadas con su instalación en extremidades superiores y a mal-posición de su punta. El contar con protocolos de atención claros, con seguimiento y control estricto de ellos, que incorporen las nuevas evidencias y capacitación sistemática del personal, son fundamentales para el uso exitoso de estos catéteres y evitar sus posibles complicaciones.

\section{Responsabilidades Éticas}

Protección de personas y animales: Los autores declaran que los procedimientos seguidos se conformaron a las normas éticas del comité de experimentación humana responsable y de acuerdo con la Asociación Médica Mundial y la Declaración de Helsinki.
Confidencialidad de los datos: Los autores declaran que han seguido los protocolos de su centro de trabajo sobre la publicación de datos de pacientes.

Derecho a la Privacidad y Consentimiento Informado: Los autores declaran que la información ha sido obtenida de datos previos en forma anonimizada, por lo cual el Comité de Ética de Investigación en uso de sus facultades, ha eximido de la obtención de un consentimiento informado, lo cual consta en el acta respectiva.

\section{Conflicto de intereses}

Los autores declaran no tener conflicto de intereses.

\section{Financiamiento}

Fondos del Departamento de Neonatología Pontificia Universidad Católica de Chile.

\section{Agradecimientos}

Al equipo de enfermeras que participó en la inserción y seguimiento de los CVCIP. A Jeannette Galleguillos y Lorena Solis, enfermeras que participaron en las primeras etapas del estudio. A la PhD María Teresa Urrutia, Profesora Asociada, Escuela de Enfermería PUC quien colaboró en la redacción del manuscrito.

\section{Referencias}

1. Stoll BJ, Hansen NI, Bell EF, et al. Trends in care practices, morbidity, and mortality of extremely preterm neonates, 1993-2012. JAMA 2015;314:1039-51.

2. D’Apremont I, Marshall G, Musalem C, et al. Trends in Perinatal Practices and Neonatal Outcomes of Very Low Birth Weight Infants during a 16-year Period at NEOCOSUR Centers. J Pediatr. 2020;225:44-50.

3. Kattan J, González A, Becker P, et al. "Survival of newborn infants with severe respiratory failure before and after establishing an ECMO program". Pediatr Crit Care Med. 2013;14:876-83.

4. Puntis JWL. Percutaneous insertion of central venous feeding catheters. Arch Dis Child .1986;61:1138-40.

5. Reynols J. Comparison of percutaneous venous catheters and teflon catheters for intravenous therapy in neonates. Neonatal Netw. 1993;12:33-41.

6. O' Grady N, Alexander M, Burns L, et al Guidelines for the prevention of intravascular catheter-related infections. Am J Infect Control 2011;39(S1):1-34.

7. Mutlu M, Aslan Y, Kul S, et al. Umbilical venous catheter complications in newborns: a 6-year single-center experience. J Matern Fetal Neonatal Med. 2015;140:1-6.

8. Kim JH, Lee YS, Kim SH, et al. Does umbilical vein catheterization lead to portal venous thrombosis? Prospective US evaluation in 100 neonates. Radiology 2001;219:645-50.

9. Pérez ME, Faunes M, Avaca M, et al. Uso de catéter central insertado periféricamente por vía percutánea en Recién Nacido. Pediatría al día 2002;18:27-31.

10. Barría M, Santander G. Cateterismo venoso central de inserción periférica en recién nacidos de cuidado intensivo. Rev Chil Pediatr .2006; 77(2):139-46.

11. Fajurí P, Pino P, Castillo A. Catéter central de inserción periférica (PICC) en pediatría. Rev Chil Pediatr. 2012;83(4):352-57.

12. Hoang V, Sills J, Chandler M, et al. Percutaneously inserted central catheter for total parenteral nutrition in neonates: Complications rates related to upper versus lower extremity insertion. Pediatrics. 2008;121(5):1152-59.

13. Paulson P, Miller K. Neonatal Peripherally inserted central catheters: Recomendations for prevention of insertion and postinsertion complications. Neonatal Netw. 2008;27(4):245-27.

14. Tomazi A, Chollopetz da Cunha M. Adverse events related to the use of central venous catheters in hospitalized newborns. Rev.Latino-Am Enfermagem. 2010;18(2):196-202.

15. Levy I, Bendet M, Samar Z, et al. Infectious complications of peripherally inserted central venous catheters in children. Pediatr Infect Dis J. 2010;29:426-9.

16. Nadroo A, Lin J, Green R, et al. Death as a complication of peripherally inserted central catheters in neonates. J Pediatr. 2000;138:599-601.

17. Sertic AJ, Connolly BL, Temple MJ, et al. Perforations associated with peripherally inserted central catheters in a neonatal 
population. Pediatr Radiol. 2018;48:10919.

18. Pet GC, EickhoffJC, McNevin KE, et al. Risk factors for peripherally inserted central catheter complications in neonates. J Perinatol. 2020;40:581-8.

19. Otaíza F, Bustamante R, Sánchez P. Informe de Vigilancia de Infecciones Asociadas a la Atención de Salud 2012. Departamento de Calidad y Seguridad del paciente. Programa control de IAAS. Ministerio de Salud, Chile. 30-2. Disponible en: https:// www.minsal.cl/sites/default/files/files/ informeinfeccionesChile2012.pdf. Acceso $20 / 11 / 2020$

20. Sengupta A, Lehmann C, Diener-West M, et al. Catheter duration and risk of CLABSI in neonates with PICCs. Pediatrics 2010;125:648-53.

21. Milstone AM, Reich NG, Advani $S$, et al. Catheter Dwell Time and CLABSIs in Neonates With PICCs: A Multicenter Cohort Study. Pediatrics 2013;132;e1609-e1615.

22. Leistner R, Piening B, Gastmeier P, et al. Nosocomial infections in very low birthweight infants in Germany: current data from the National Surveillance
System NEO-KISS. Klin Padiatr. 2013; 225:75-80.

23. Chien LY, Macnab Y, Aziz K, et al; Canadian Neonatal Network. Variations in central venous catheter-related infection risks among Canadian neonatal intensive care units. Pediatr Infect Dis J. 2002;21:505-11.

24. Dubbink-Verheij GH, Bekker V, Pelsma ICM, et al. Bloodstream Infection Incidence of Different Central Venous Catheters in Neonates: A Descriptive Cohort Study. Front Pediatr. 2017;5:142. doi: 10.3389/fped.2017.00142

25. Njere I, Islam S, Parish D, et al. Outcome of peripherally inserted central venous catheters in surgical and medical neonates. J Pediat Surg. 2011;46:946-50.

26. Urzua S, Ferres M, Garcia P, et al. Estrategias para reducir infecciones, uso de antibióticos y sus efectos en una unidad de neonatología. Rev Chilena Infect. 2017;34:99-117.

27. Oleti T, Jeeva Sankar M, Thukral A, et al. Does ultrasound guidance for peripherally inserted central catheter (PICC) insertion reduce the incidence of tip malposition? - a randomized trial. J Perinatol. 2019;39:95-101.
28. Trotter C. Inadvertent Catheterization of the Ascending Lumbar Vein. Neonatal Netw. 2009; 28:179-83.

29. Romaniello H, Alda E. Localización epidural de un catéter percutáneo venoso transfemoral izquierdo. Arch Argent Pediatr. 2004;102:287-9.

30. Bashir RA, Swarnam K, Vayalthrikkovil $\mathrm{S}$, et al. Association between Peripherally Inserted Central Venous Catheter Insertion Site and Complication Rates in Preterm Infants. Am J Perinatol. 2016;33:945-50.

31. Elmekkawi A, Maulidi H, Mak W, et al. Outcomes of upper extremity versus lower extremity placed peripherally inserted central catheters in a medicalsurgical neonatal intensive care unit1. J Neonatal Perinatal Med. 2019;12:57-63.

32. Uslu S, Ozdemir H, Comert S, et al. The effect of low-dose heparin on maintaining peripherally inserted percutaneous central venous catheters in neonates. J Perinatol. 2010;30:794-99.

33. Bin-Nun A, Wasserteil N, Nakhash R, et al. Heparinization of Long Indwelling Lines in Neonates: Systematic Review and Practical Recommendations. IMAJ 2016;18:692-96. 